\title{
Evaluation of an Ultrasound-Guided Focused Ultrasound CMUT Probe for Targeted Therapy Applications
}

\author{
Dominique Gross ${ }^{1}$, Mathieu Legros ${ }^{1}$, Philippe Vince², Dominique Certon ${ }^{3}$ \\ ${ }^{1}$ VERMON SA, Tours, France \\ ${ }^{2}$ François-Rabelais University, CETU Althaïs Technologies, Tours, France \\ ${ }^{3}$ GREMAN UMR 7347 Université de Tours, CNRS, INSA Centre Val de Loire, Tours, France \\ Email: dominique.certon@univ-tours.fr
}

How to cite this paper: Gross, D., Legros, M., Vince, P. and Certon, D. (2018) Evaluation of an Ultrasound-Guided Focused Ultrasound CMUT Probe for Targeted Therapy Applications. Open Journal of Applied Sciences, 8, 25-45.

https://doi.org/10.4236/ojapps.2018.81003

Received: December 21, 2017

Accepted: January 27, 2018

Published: January 30, 2018

Copyright $\odot 2018$ by authors and Scientific Research Publishing Inc. This work is licensed under the Creative Commons Attribution International License (CC BY 4.0).

http://creativecommons.org/licenses/by/4.0/

\begin{abstract}
Capacitive Micromachined Ultrasonic Transducer (CMUT) technology, which has been widely studied in the field of medical imaging, possesses strong design flexibility due to its manufacturing process. Many applications could benefit from this unique feature, especially those that require different operating ultrasonic frequencies. This article reports on the characterization of the therapeutic low-frequency field provided by an ultrasound-guided focused ultrasound CMUT probe that is connected to a custom ultrasonic scanner for hyperthermia applications. The study begins by mapping the focused ultrasonic beam in the vicinity of the focal spot and a parametric analysis providing the maximum peak-to-peak (PTP) pressure delivered by the probe under different acoustic conditions. The measured maximum PTP pressure at the targeted operating frequency of $1 \mathrm{MHz}$ is $3 \mathrm{MPa}$, with a maximum of $3.6 \mathrm{MPa}$ at $1.25 \mathrm{MHz}$. Based on an in vitro setup found in the literature, the temperature elevation at the focal point was measured, showing results in agreement with the targeted applications ( $\max . \Delta \mathrm{T}=7.5^{\circ} \mathrm{C}$ ). The article concludes with a reliability study considering the delivered pressure and the self-heating of the CMUT probe: the results show the good stability of the pressure amplitude over $1.8 \times 10^{9}$ cycles at a duty cycle of $40 \%$, with a moderate internal heating of $3^{\circ} \mathrm{C}$.
\end{abstract}

\section{Keywords}

Capacitive Micromachined Ultrasonic Transducers, CMUT, USgFUS, Targeted Therapy 


\section{Introduction}

Alongside the well-known use of high-amplitude ultrasonic sequences for ablative purposes, the scientific community is experiencing an exciting era in the development of new therapeutic protocols, for which ultrasonic waves are exploited in a more indirect way. In particular, the targeted drug delivery field, which is being increasingly promoted to reduce drugs side effects and improve the treatment efficiency, is widely benefiting from this keen interest in therapeutic ultrasound. Among the most common applications that are still under development or close to mature exploitation, one can mention drug and gene transfection [1] [2], transdermal drug delivery [3], and the disruption of the blood-brain barrier [4]. Generally, the operating frequency is quite far below the diagnostic imaging frequency, in the range from a few tens of kilohertz up to a few megahertz, with the most commonly used region around $1 \mathrm{MHz}$ [5]. Most of these new therapeutic approaches still need deep maturation through preclinical testing on small animals.

Therapy involves prior diagnosis and scrupulous treatment guidance. Historically and for several legitimate motivations, magnetic resonance imaging (MRI) is often used to achieve these requirements. MRI provides high-quality images with significant ease of tissue heat monitoring. The development of new devices and protocols, referred to as magnetic resonance guided focused ultrasound (surgery) (MRgFUS) [6] [7], has resulted from this natural combination. However, magnetic resonance imaging is not free from drawbacks: important aspects include the cost, safety issues related to magnetic fields and ferromagnetic objects, and the dependence on the patient's condition. For these reasons, ultrasonic guidance may be more suitable in some cases: ultrasonic imaging is cheap, fast and easy to use and provides real-time images. Therefore, ultrasound guided focused ultrasound devices (USgFUS) [8] have regained the interest they had lost with the advent of MRgFUS.

For all these reasons, our group has undertaken the development of a USgFUS probe dedicated to the maturation of targeted therapy protocols on small animals. The main originality of this probe is the use of CMUT (capacitive micromachined ultrasonic transducer) arrays instead of the classical piezoelectric transducers. A CMUT transducer is a microelectromechanical chip made of hundred/thousands of microscale membranes driven by electrostatic forces. Presented for the first time in 1994 by Haller and Khuri-Yakub [9], CMUT transducers have been deeply studied for ultrasonic imaging purposes [10]-[15] until the recent first international commercialization of a CMUT linear probe [16]. Several studies have also assessed the potential benefits of CMUT arrays for therapeutic intents, but all have been strictly circumscribed to high-intensity focused ultrasound (HIFU) [17] [18] [19] [20] [21]. Additionally, these studies have generally considered only the early stage of development and characterization, whereas the complete functionality of a fully packaged CMUT-based probe for FUS applications still needs to be demonstrated. This is one of the goals of 
this paper. CMUT transducers seem to provide significant benefits over the state-of-the-art piezoelectric transducers. First, their intrinsically low mechanical losses [22] result in low spurious self-heating of the probe and thus the potential ability to execute high duty cycle (DC) therapeutic sequences without the need for an external cooling system. Furthermore, the thickness of the material fixes the operating frequency for piezoelectric transducers, making the conception of an USgFUS probe embedding two different types of transducer difficult, as the designers have to deal with different transducer sizes depending on their operating frequency. This issue is naturally exacerbated when the imaging and therapeutic frequencies are quite far from each other. Significant developments based on this approach have nevertheless been reported in the literature, with promising results [23] [24] [25]. Sometimes, USgFUS designers prefer to use so-called "dual-mode transducers", which often means to focus on one of the two modalities at the expense of the other: for instance, [8] primarily designed their USgFUS probe with therapeutic considerations, and methods were later investigated to obtain satisfying images [26]. Nevertheless, these solutions are only applicable to situations in which the imaging and therapeutic frequencies are quite close to each other to deal with the relatively narrow bandwidth of piezoelectric transducers. On the other hand, CMUT transducers are much more flexible in this respect because of their well-known large bandwidth and the possibility of easily incorporating two completely different types of transducer side by side by simply scaling the size of the membranes. Furthermore, as they are based on microelectromechanical systems, CMUT transducers provide an extremely high degree of compactness and packaging. Therefore, high-end probes including multi-frequency compact arrays are achievable using CMUT technology, which are highly beneficial to the development of breakthrough USgFUS products. The USgFUS CMUT probe we have developed has been previously presented [27]. Contrary to the previous article that was dedicated to the probe description and the very first characterizations of the CMUT arrays, this article is devoted to the in-depth characterization of the therapeutic ultrasonic beam and the assessment of the CMUTs viability for hyperthermia applications.

\section{Materials and Methods}

\subsection{Overview of the USgFUS CMUT Probe}

The design of the fabricated USgFUS probe (Figure 1(a)) was driven by two considerations. The first is clearly the acoustic requirements of the target applications. In this study, a frequency of $1 \mathrm{MHz}$ was chosen because of its use in numerous therapeutic treatments [5]. More precisely, sonoporation and the triggered release of thermosensitive liposomes (TSLs) [28] were targeted. Whereas sonoporation generally requires a relatively low peak negative pressure (PNP), the mild hyperthermia required to reach the melting phase transition temperature of the TSLs is more challenging to generate at $1 \mathrm{MHz}$ : [29] showed that this temperature, typically approximately $42^{\circ} \mathrm{C}$, can be reached at $1 \mathrm{MHz}$, 


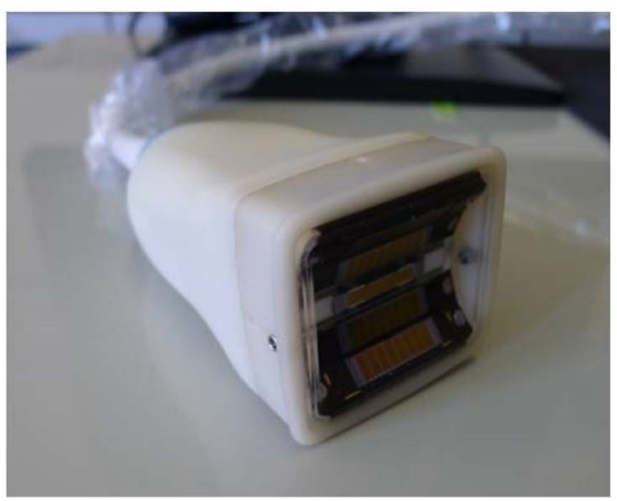

(a)

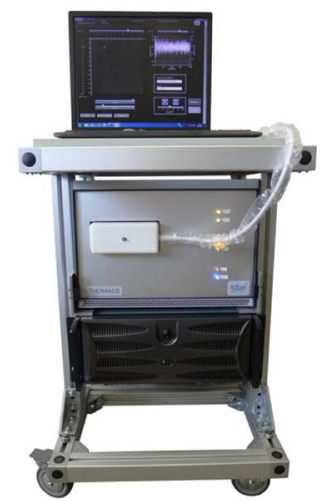

(b)

Figure 1. Fabricated USgFUS probe (a); custom US scanner (b).

40\% DC and a $1 \mathrm{kHz}$ pulse repetition frequency (PRF), with a minimum PNP of 1.25 $\mathrm{MPa}$ (or 2.5 MPa PTP). The second critical aspect is that working on small animals involves a small exploration depth, which requires the acoustic intensity to be concentrated very close to the transducer. For these reasons, a mechanically focused topology has been designed, as depicted in Figure 2. The probe is made of 5 linear arrays placed on an arc of a circle with a radius of $20 \mathrm{~mm}$. Except for the operating frequencies, this design is very similar to the one proposed by Stephens et al. [23] [30]. The central array is a linear array of 128 elements centered at $16 \mathrm{MHz}$ (high frequency, HF) that is used for imaging, and it is surrounded on each side by two low-frequency (LF) linear arrays of 8 elements dedicated to the therapeutic beam. Additional information about the CMUT transducers are reported in Table 1. Note that we chose to operate the therapeutic CMUT transducers in a rarely used mode, i.e., far below their center frequency in the pseudo-static regime [31]. Indeed, designing CMUT cells having a very low center frequency would lead to very large membranes and thus significant static deflection due to the atmospheric pressure. Static deflection involves a decrease of the actual gap height available for membrane displacement. As the pressure output is linearly proportional to the average dynamic displacement amplitude of the membranes, strongly deflected membranes would produce weak pressure amplitudes. Therefore, a trade-off was made by choosing a CMUT cell size of $40 \mu \mathrm{m} \times 40 \mu \mathrm{m}$, which leads to a center frequency of approximately $3 \mathrm{MHz}$. Note that impedance matching circuits were embedded in the probe body, consisting of $4.7 \mu \mathrm{H}$ inductances connected in parallel with each LF emitter. Their role is to decrease as much as possible the imaginary part of the device electrical impedance to increase the power transmitted from the emitters to the CMUT probe through the cable harness (approximately $1.5 \mathrm{~m}$ length). Finally, all of the transducers were coated with a $300 \mu \mathrm{m}$ silicone rubber coating as passivation, with an attenuation coefficient of $0.2 \mathrm{~Np} \cdot \mathrm{mm}^{-1}$ at $3.5 \mathrm{MHz}$ and an acoustic impedance of 1.32 MRayl.

More detailed information about the design stage, the manufacturing process and the choice of the CMUT geometry are given by [27]. 


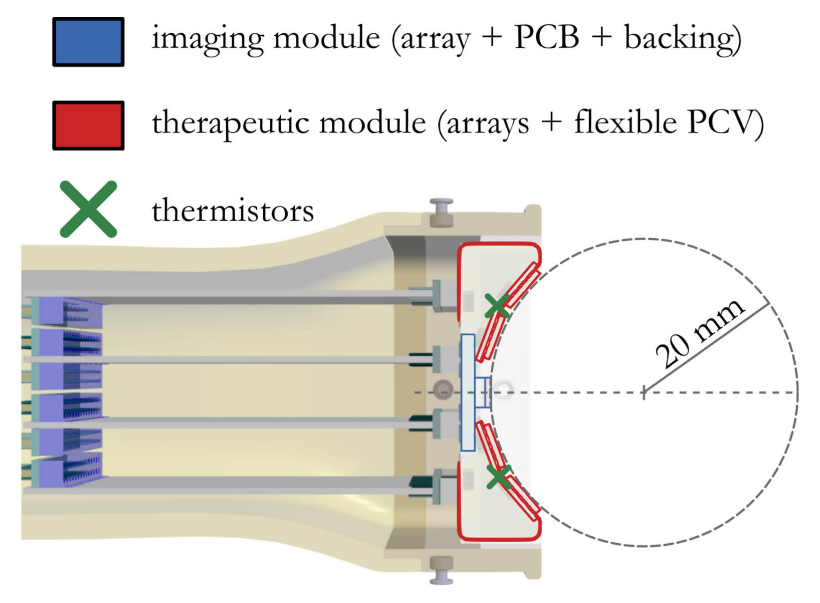

Figure 2. Representation of the CMUT probe dual topology.

Table 1. Low Frequency (LF) and High Frequency (HF) arrays metrics.

\begin{tabular}{ccc}
\hline & LF & HF \\
\hline Elements & 8 & 128 \\
Elevation $[\mathrm{mm}]$ & 5 & 2.8 \\
Pitch $[\mu \mathrm{m}]$ & 2308 & 125 \\
Membrane $[\mu \mathrm{m} \times \mu \mathrm{m}]$ & $40 \times 40$ & $15 \times 21$ \\
Gap height $[\mathrm{nm}]$ & 500 & 100 \\
Membranes thickness $[\mathrm{nm}]$ & & 800 \\
\hline
\end{tabular}

\subsection{Description of the Dedicated US Scanner}

Alongside the development of the probe, a dedicated US scanner that was specially designed to deliver FUS sequences and imaging feedback has been manufactured (Figure 1(b)). A scheme describing the whole architecture of the scanner is reported in Figure 3. In red is drawn the electronic circuitry dedicated to High Frequency imaging, whereas in blue is represented the one related to LF emission. There is one master FPGA that controls each FPGA embedded on each HF board through a cPCI backplane. A PC controller drives the platform through an USB link and receives RF signals from the beamforming unit.

The two modalities can be used independently or simultaneously using specific software. The latter is split into three windows, one for each modality and one setting window to set the electrical and acoustical parameters. On the imaging side-which is not extensively described here because it is outside the scope of this article (more information in [32]) - the reconstructed B mode image is displayed with the classical setting parameters (contrast, brightness, gain, specific RF line display). The beamforming process is a classical delay-and-sum, with tunable frequency, transmit and receive apertures.

The current scanner manages 64 HF imaging channels but can be extended to 128 channels by duplicating the electronic Tx/Rx boards. The HF 64 transmitting boards are able to produce 3 -state coded pulses on 64 independent and 


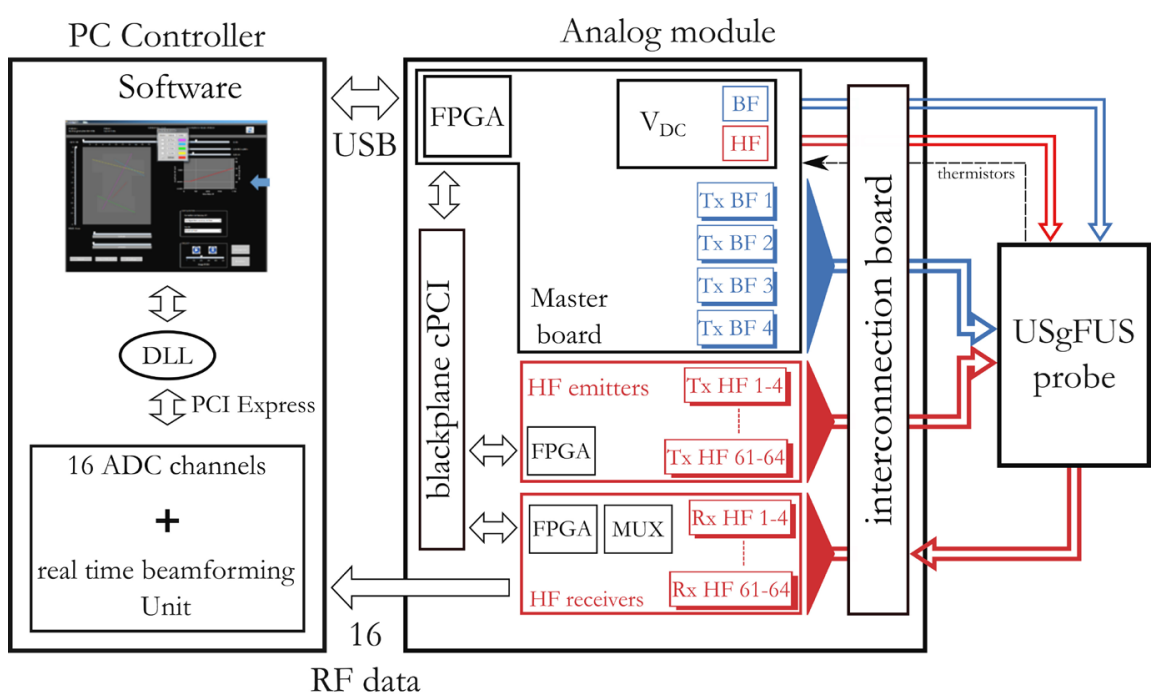

Figure 3. Scheme of the US scanner architecture.

rigorously synchronous channels. The HF receiving boards contain 16 amplifying channels (100 MHz-bandwidth) with a programmable gain up to $40 \mathrm{~dB}$, associated with a 4:1 multiplexer to address all the receivers. The real-time beamforming unit is made of five field-programmable gate arrays (FPGA) and a RAM block to numerically construct the RF signals. Ultrafast links of $1 \mathrm{~Gb} / \mathrm{s}$ are used to transfer the 16 digitized signals from the ADC module to the beamforming unit. The master board embeds the LF transmitters for therapeutic purposes, as well as the DC power supplies required to bias the CMUT cells.

On the FUS side, 4 different channels are dedicated to the FUS emission, thus enabling the use of electronic focusing. Note that this relatively low number of focusing channels naturally influenced the probe design, especially the need for mechanical focusing. The scanner can produce long sinusoidal burst signals (max. 50\% DC, $100 \mathrm{~Hz}$ to $10 \mathrm{kHz}$ PRF) with an emitting frequency tunable from $500 \mathrm{kHz}$ to $2 \mathrm{MHz}$ and an expected maximum PTP voltage of $80 \mathrm{~V}$. This scanner was used during all of the measurements presented thereafter.

\subsection{Self-Heating Assessment}

The self-heating of the CMUT transducers was assessed by two thermistors embedded into the probe. Each of them is inserted into the silicon coating, between the low-frequency transducers (see Figure 2). At a given time, the temperature is measured through the resistance value of the two thermistors. At this step of the development, the temperature variations were recorded and stored manually. In order to follow the temperature variation against time, the internal clock of the computer was used to increment at the right value the sampling times.

\subsection{Hydrophone Measurement}

The majority of the characterizations reported hereafter were based on a hydrophone setup. The probe was placed in front of a water tank where a specific 
opening was created in order that only the front face of the probe is in contact with the fluid (to avoid any electric damages that could be caused by water infiltration). A hydrophone (HGL-0085, Onda Corp., Sunnyvale, CA, USA) was mounted on a motorized positioning stage to allow the automatic mapping of the pressure field through a MATLAB (The MathWorks, Inc., Natick, MA, USA) script. The averaging of the acquired signal may be employed on the connected oscilloscope (WaveRunner 104Xi, LeCroy, Chestnut Ridge, NY, USA) to lower the noise threshold. This hydrophone is calibrated from $250 \mathrm{kHz}$ up to $40 \mathrm{MHz}$ and is directly connected to a $20 \mathrm{~dB}$ preamplifier (AH-2010, Onda Corp., Sunnyvale, CA, USA) calibrated up to $100 \mathrm{MHz}$. The recorded voltage signals are automatically converted to pressure signals by a MATLAB script.

\subsection{Simulation}

To provide a baseline to assess the quality of the whole probe with respect to the designed one, the measured pressure fields were compared with the simulated ones. The goal is to compare the spatial distributions of the pressure fields but not the absolute pressure amplitude because we had no significant foreknowledge of the average displacement of the membranes.

To this end, the free open-source DREAM (Discrete REpresentation Array Modelling) toolbox [33] [34] [35] was used in the MATLAB environment. This toolbox allows the computation of pressure fields radiated by arbitrarily shaped acoustic sources under the hypothesis of linear propagation: the model provides the spatial impulse response of the acoustic device, which can be convoluted afterward with the sources particular acceleration (second time derivative of the displacement). In this study, this latter was made of 10 cycles of a sine wave, centered at $1 \mathrm{MHz}$. As the acoustic attenuation in water is very weak and it unreasonably extends the computing time, all the simulations were performed without attenuation. Each one of the 32 elements that comprise the FUS part of the probe was simulated as a rectangular piston source, and the silicone coating that covers the transducers was neglected. The computer used was a HP Z400 Workstation with 16 Go RAM and a W3680 3.33 GHz Intel Xeon CPU.

\subsection{Temperature Elevation Measurement}

To measure the temperature elevation produced by the probe at the focal spot, a setup adapted from the one proposed by [36] was used. A thermocouple (Dostmann Electronic, Wertheim-Reicholzheim, Germany) was immersed into a plastic cuvette (Fischer Scientific, Illkirch, France) filled with a tissue-like absorptive fluid, i.e., glycerol (3.6 Np/(mMHz) [36]). The thermocouple was then precisely placed in the vicinity of the focal spot using the imaging feedback provided by the probe (see Figure 4 ). The temperature elevation was produced by a long burst exposure (40\% - 50\% DC, $1 \mathrm{kHz}$ PRF, $V_{D C}=90-110 \mathrm{~V}$, command value of $V_{A C}=60-80 \mathrm{~V}_{p p}$ ) for 10 minutes. An acoustic window made of thin Mylar film $(15 \mu \mathrm{m})$ was chosen to minimize the disruption of the FUS beam. Because the 


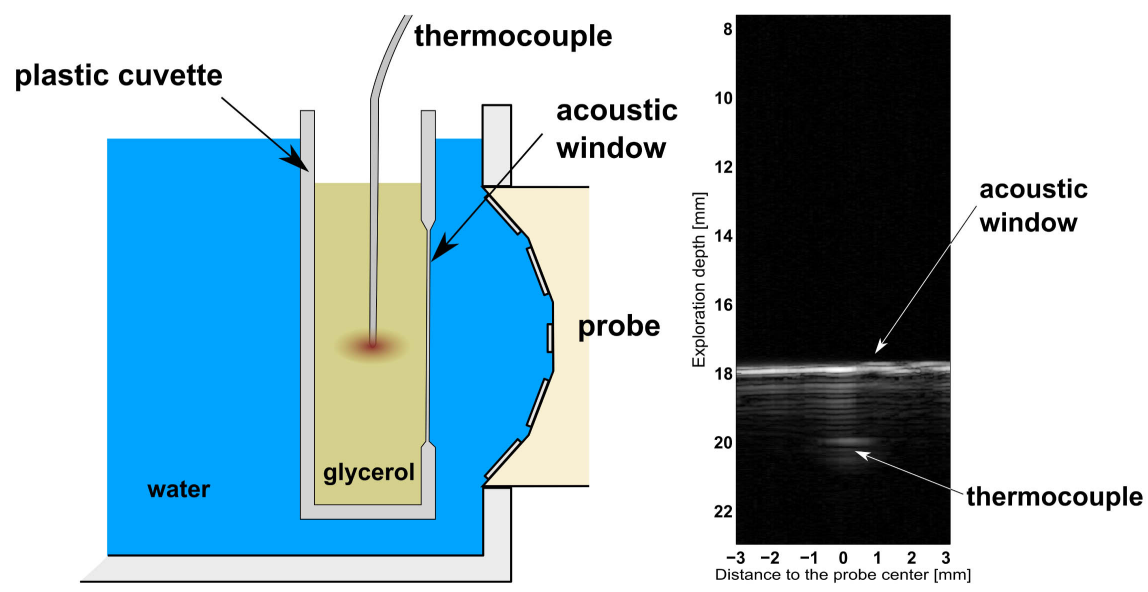

Figure 4. Temperature elevation setup and B-mode image of the thermocouple located at the focal distance.

thermocouple was not programmable, the temperature values were manually stored. As for the measure of temperature inside the probe, the sampling time of the temperature was incremented by using the internal clock of the computer.

\section{Results}

\subsection{Validation of the Pressure Field}

The simulated and measured pressure mappings of the FUS beam are reported in Figure 5, with and without the use of the electronic focusing provided by the US scanner. The mapping was performed in three mutually perpendicular planes, crossing at the targeted focal point (i.e. $20 \mathrm{~mm}$ ). For comparison purposes, the mesh in the simulation matches the one used for the measurement (a total of 1323 meshing nodes with a step of $0.5 \mathrm{~mm}$ in all directions). Each recorded signal was averaged 20 times.

As this measurement took a lot of time, we chose not to overtax the probe by using demanding excitation voltages: a bias voltage of $V_{D C}=80 \mathrm{~V}$ with a command value for the signal amplitude of $V_{A C}=60 \mathrm{~V}(1 \mathrm{MHz}, 10$ cycles of sine wave, $1 \mathrm{kHz}$ PRF) was delivered by the US scanner. Therefore, no quantitative value of the pressure amplitude is provided with this measurement, which explains why the pressure fields displayed in Figure 5 are normalized by the maximum pressure recorded for both the simulation and measurement.

Figure 5 clearly highlights the good matching between the expected pressure fields and the measured ones. The size of the focal spot is an important feature for therapeutic applications. Because of the strong focusing gain of the probe, the accurate measurement of the focal spot would require a fine meshing and the perfect positioning and alignment of both the probe and the hydrophone. For these reasons, and because the simulated pressure fields are very close to the measured ones, we choose to estimate the volume of the focal spot from the simulated data: the spatial discretization step was decreased to $0.25 \mathrm{~mm}$ in the and azimuth, and the pressure field was computed in a volume centered around the 


\section{with electronic focusing at $20 \mathrm{~mm}$}
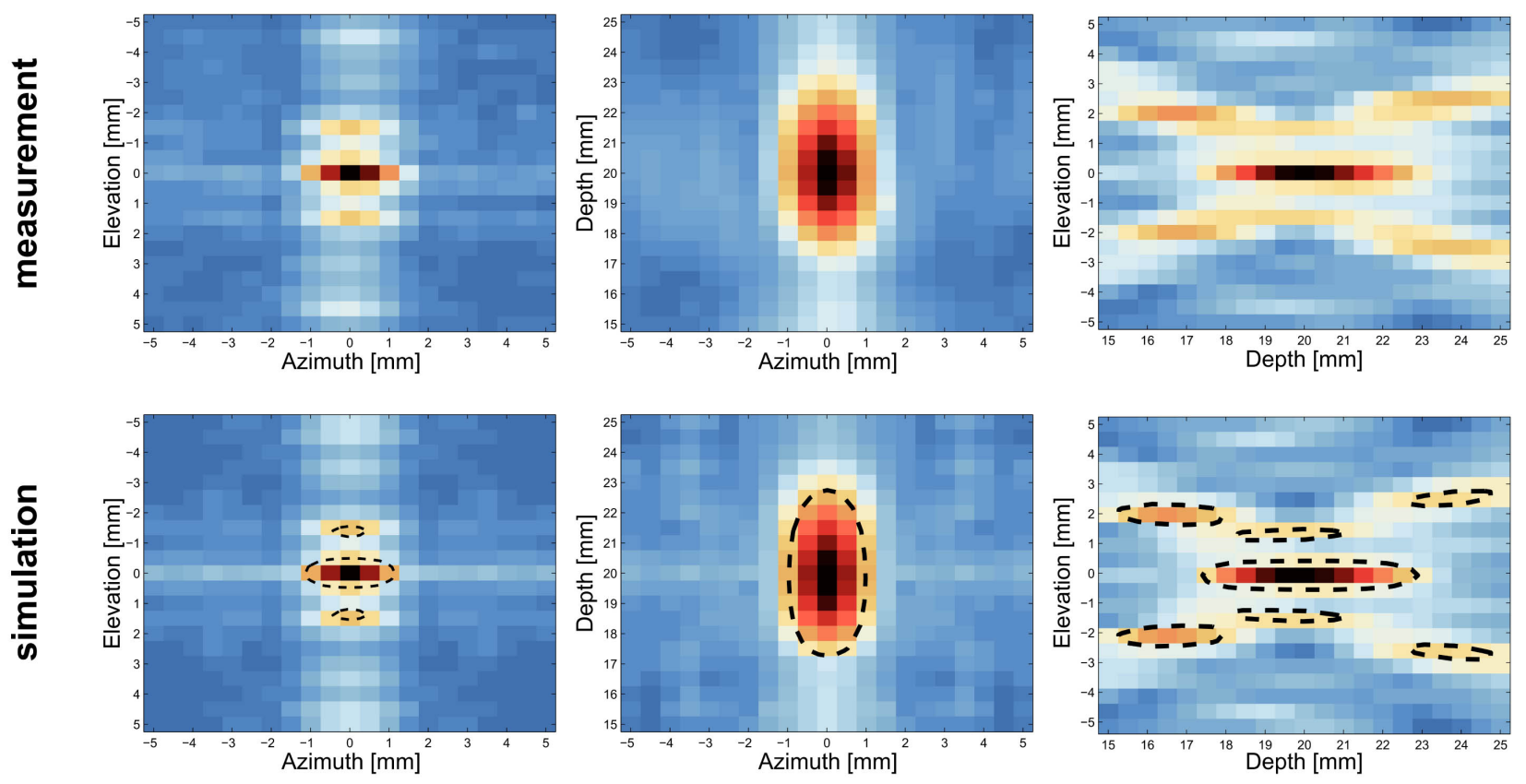

without electronic focusing
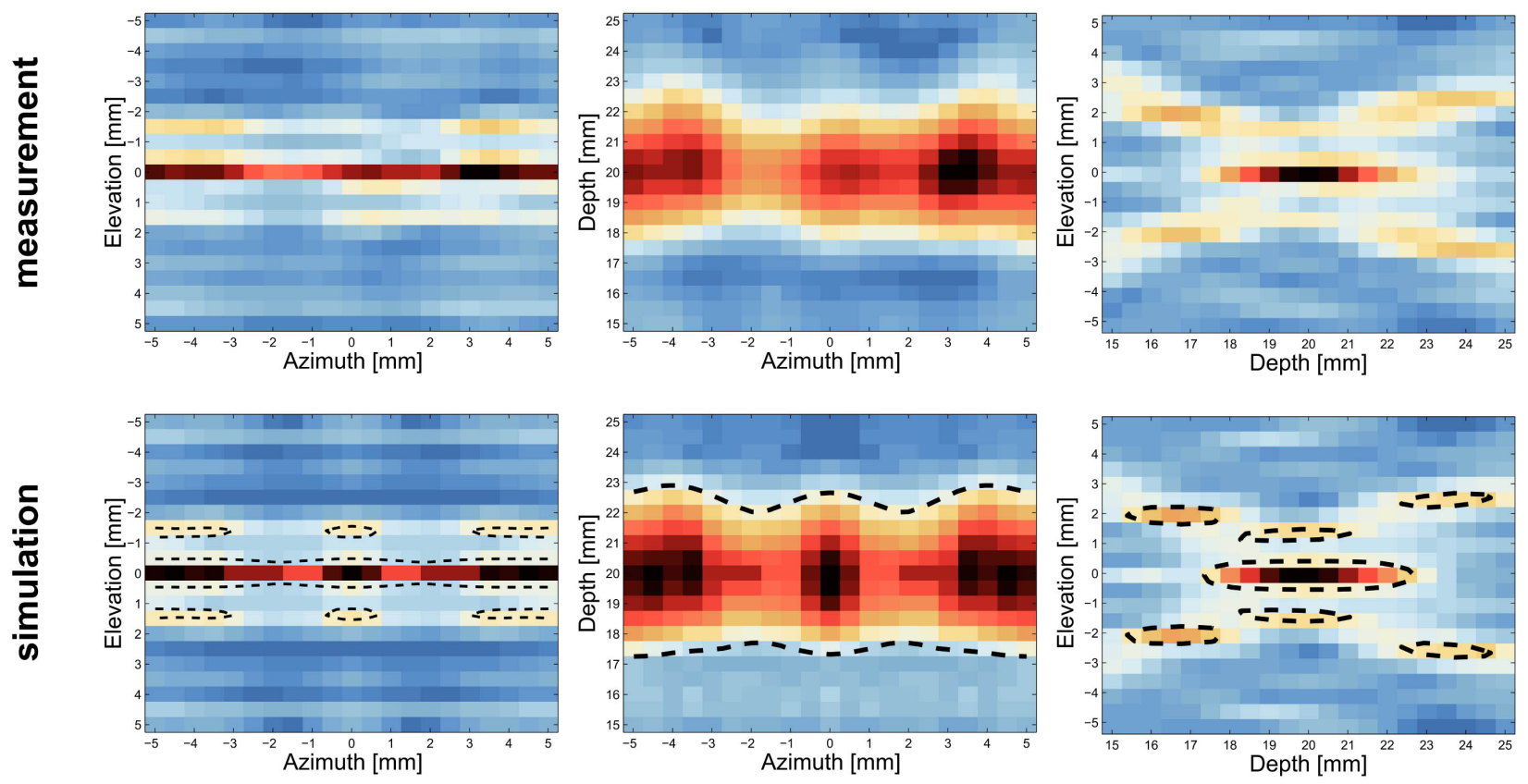

$\min$

Pressure (a.u.)

$\max$

Figure 5. Pressure field mapping (measurement and simulation) with and without electronic focusing. The dash line on simulated graphs delimits the half maximum pressure isoline (the focal spot).

focal spot (elevation: from $-10 \mathrm{~mm}$ to $10 \mathrm{~mm}$; azimuth: from $-10 \mathrm{~mm}$ to 10 $\mathrm{mm}$; depth: from $14 \mathrm{~mm}$ to $25 \mathrm{~mm}$ ), leading to a computation at 150,903 nodes. Then, the focal spot volume was estimated by summing the number of voxels 
$\left(\mathrm{dV}=\mathrm{dx} \times \mathrm{dy} \times \mathrm{dz} \approx 0.03 \mathrm{~mm}^{3}\right)$ having a pressure magnitude greater than or equal to half the maximum pressure (isolines at half the maximum pressure are represented by the dashed lines in Figure 5). Thereby, the focal spot volume was estimated to be $18.5 \mathrm{~mm}^{3}$ and $205 \mathrm{~mm}^{3}$ with and without electronic focusing, respectively.

\subsection{Variation of the Pressure Amplitude as a Function of $V_{A C} / V_{D C}$}

After the validation of the pressure field, the major challenge of characterizing the therapeutic US beam was naturally the quantification of the maximum pressure delivered by the probe and thereby its ability or lack thereof to meet the requirements imposed by the two target applications. For this purpose, the hydrophone was carefully placed in the focal spot to look for the maximum output signal, and we recorded the maxima for various command values of PTP $V_{A C}$ (from $10 \mathrm{~V}$ to $80 \mathrm{~V}$ with a step of $10 \mathrm{~V}$ ) and $V_{D C}$ (from $10 \mathrm{~V}$ to $110 \mathrm{~V}$ with a step of $10 \mathrm{~V}$ ) voltages. The input signal was still made of 10 cycles of a sine wave at 1 $\mathrm{MHz}, 1 \mathrm{kHz}$ PRF. The results are reported in Figure 6, with and without the

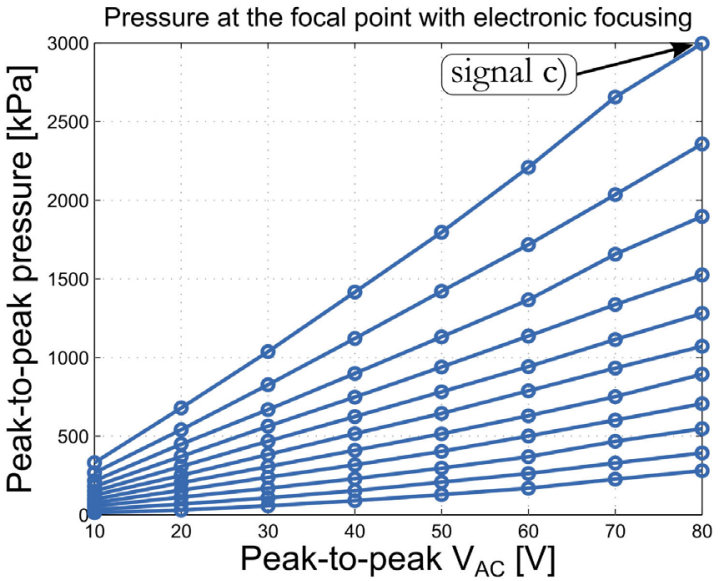

(a)

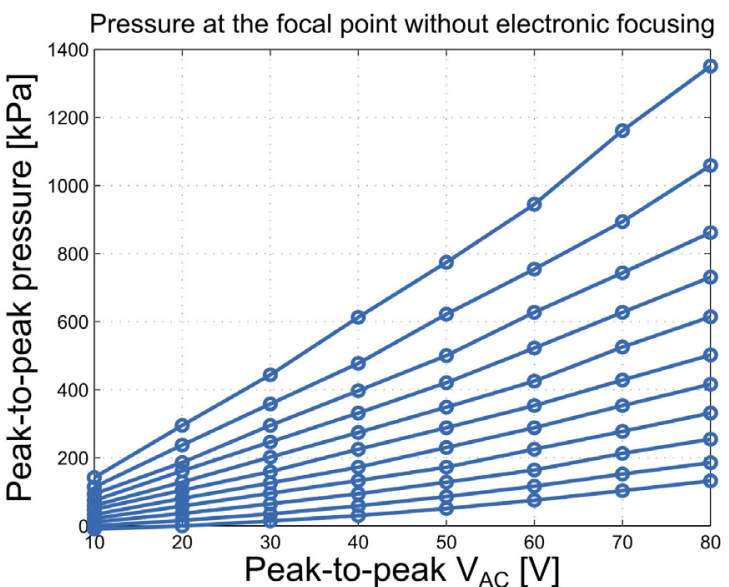

(b)

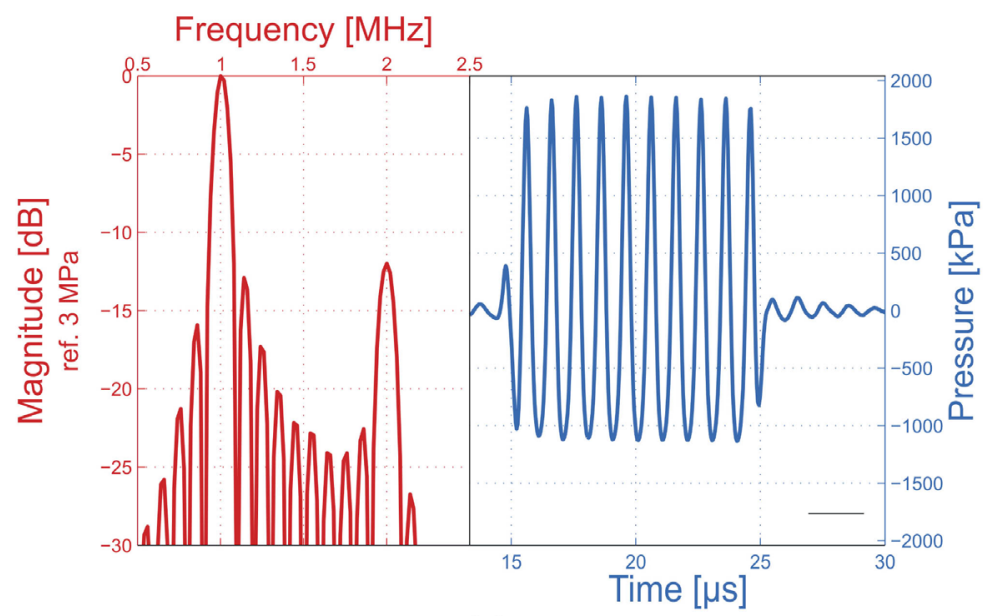

(c)

Figure 6. Measurement of the maximum delivered pressure for various $V_{A C}$ and $V_{D C}$ voltages, with (a) and without (b) the use of the electronic focusing. Time and frequency representation of the maximum measured pressure signal (c). 
use of the electronic focusing, as well as the maximum pressure signal we recorded. With these excitation conditions, the maximum PTP pressure amplitude delivered by the probe reaches $3 \mathrm{MPa}$, with a maximum PNP of $1.15 \mathrm{MPa}$. The pressure signal is strongly nonlinear, with a second harmonic approximately $-12.5 \mathrm{~dB}$ below the fundamental one, as expected by using the CMUT cells below their center frequency. This behavior is well-known and has already been reported in the literature [31] [37]. Without the use of the electronic focusing, the maximum PTP pressure is $1.4 \mathrm{MPa}$, with a PNP amplitude of $540 \mathrm{kPa}$.

\subsection{Variation of the Maximum Pressure with the Frequency}

Before the use of the probe with actual emitting sequences, we measured, at the focal point, the maximum pressure amplitude as a function of the ultrasonic frequency. To this end, the hydrophone was set in the focal spot, and $V_{A C}$ and $V_{D C}$ were set to the values that previously delivered the maximum pressure amplitude, i.e. $80 \mathrm{~V}$ (maximum expected PTP amplitude delivered by the US scanner) and $110 \mathrm{~V}$, respectively. The pressure amplitude was then recorded, as well as the effective emitter $V_{A C}$ (measured at the emitters output).

10 cycles of a sine wave with a $1 \mathrm{kHz}$ PRF were still used, and the ultrasonic frequency was swept from $800 \mathrm{kHz}$ to $1.3 \mathrm{MHz}$. Outside this frequency range, the pressure amplitude rapidly collapses because of the electrical components bandwidths and narrow impedance matching. The results are reported in Figure 7. As one can note, the optimal emitting frequency is approximately $1.25 \mathrm{MHz}$, with an effective PTP emitter $V_{A C}$ of $87 \mathrm{~V}$, a PTP of $3.6 \mathrm{MPa}$ (PNP 1.5 MPa), and a more linear ultrasonic signal (second harmonic at $-16 \mathrm{~dB}$ ).

\subsection{Mild-Hyperthermia at the Focal Point}

Once the plain functionality of the therapeutic US module has been demonstrated, the probe was tested under the actual insonification sequences required by the target applications. The goal of this measurement was to assess its ability to increase the temperature in glycerol. The temperature elevations recorded by the thermocouple in the vicinity of the focal spot are reported in Figure 8 for different insonification sequences. The temperature elevation threshold required to reach the liposomes melting temperature at body temperature is displayed as a dashed black line.

For all of the tested insonification sequences, the results clearly show the presence of an ultrasonic pressure field sufficient to induce thermal effects in the glycerol. Two emitting conditions allow the temperature elevation required to melt TSLs to be achieved within approximately two minutes of insonification. These are the conditions under which the US scanner delivers the maximum voltage amplitudes, with a duty cycle between $40 \%$ and $50 \%$ and are in agreement with the acoustic parameters we targeted during the conception of the CMUT probe. The maximum temperature elevation the probe induced in glycerol is $7.5^{\circ} \mathrm{C}$. Note that even without electronic focusing, the temperature 


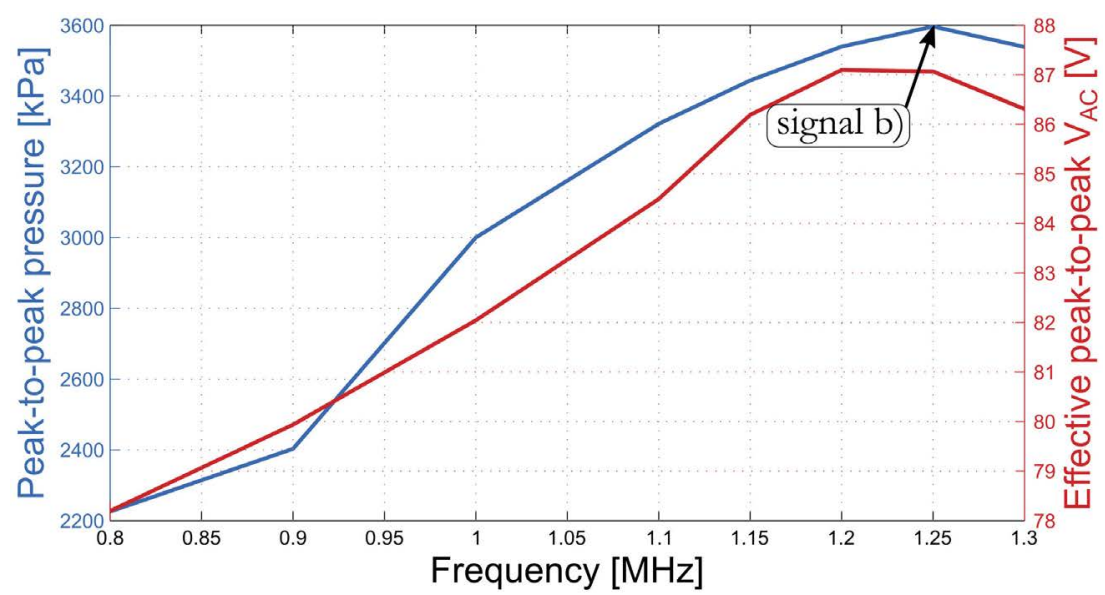

(a)

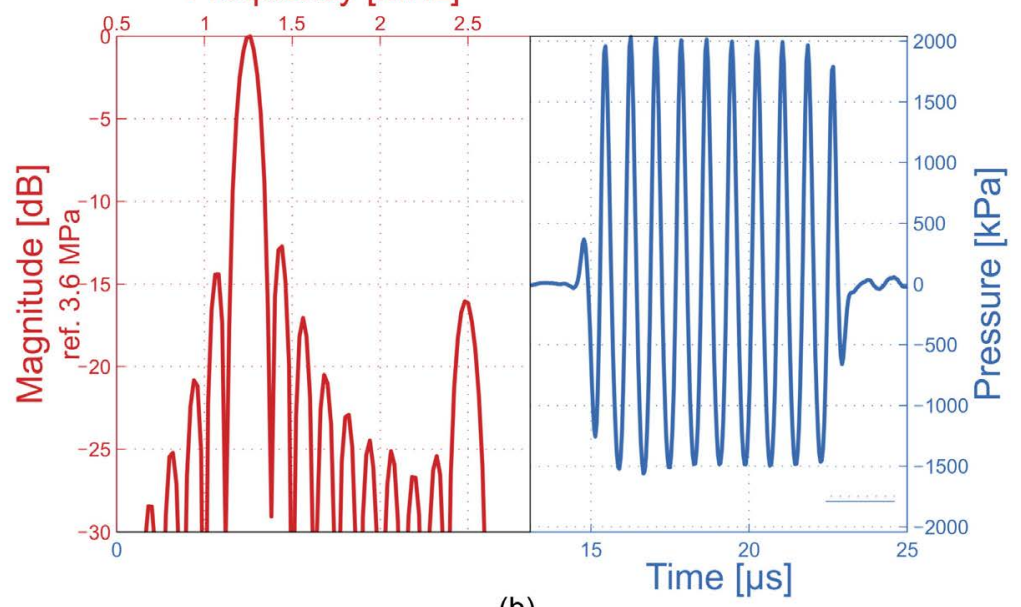

(b)

Figure 7. (a) Measurement of the maximum delivered pressure (blue line) and effective emitters $V_{A C}$ (red line) for different ultrasonic frequencies; (b) Maximum pressure signal recorded on the overall frequency range.

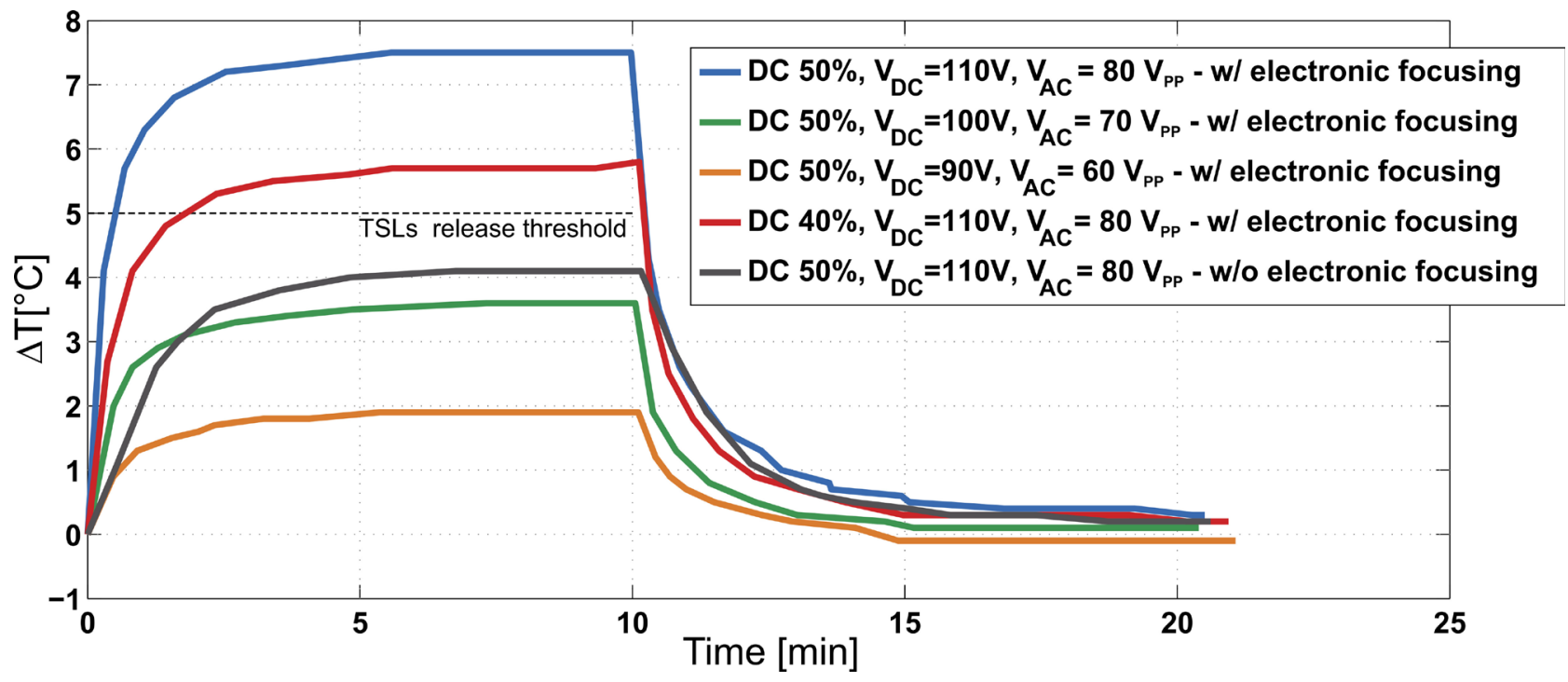

Figure 8. Temperature elevation in the vicinity of the focal spot for different insonification sequences. 
elevation at the focal spot is $4^{\circ} \mathrm{C}$ under the most intense insonification sequence the US scanner can currently provide.

\subsection{Preliminary Study of Reliability}

Different from the imaging short pulses, the long burst insonification sequences required by therapeutic applications are very demanding of the transducers. For these reasons, it is necessary to verify the viability of the employed technology for a relatively long duration. Potential failures may be engendered by charges trapped in the CMUT dielectric layers, thermal effects, or mechanical strain. For these reasons, the probe reliability was checked for 100 minutes of intermittent therapeutic exposure ( 15 minutes of emission and 5 minutes without emission, repeated 5 times) to mimic actual operation. The CMUT transducers were excited with a command value of $V_{A C}=80 \mathrm{~V}_{\mathrm{pp}}, V_{D C}=100 \mathrm{~V}, 40 \% \mathrm{DC}, 1 \mathrm{kHz}$ PRF, at $1 \mathrm{MHz}$. This represents $1.8 \times 109$ displacement cycles and a cumulative actuation time of 30 minutes. Three different parameters were simultaneously analyzed: the robustness of the US scanner by recording the delivered $V_{A C}$ amplitude, the robustness of the probe by recording the delivered pressure, and the internal self-heating through the embedded thermistors. To avoid any damage to the hydrophone, which is not suitable for long burst measurements, it was placed far away from the focal spot and out of the probe axis to lower the total energy deposited on the tip. Therefore, only the pressure amplitude variation was investigated to assess the stability of our probe, and the results are reported in Figure 9. The pressure and voltage amplitudes are normalized by their respective maximum values.

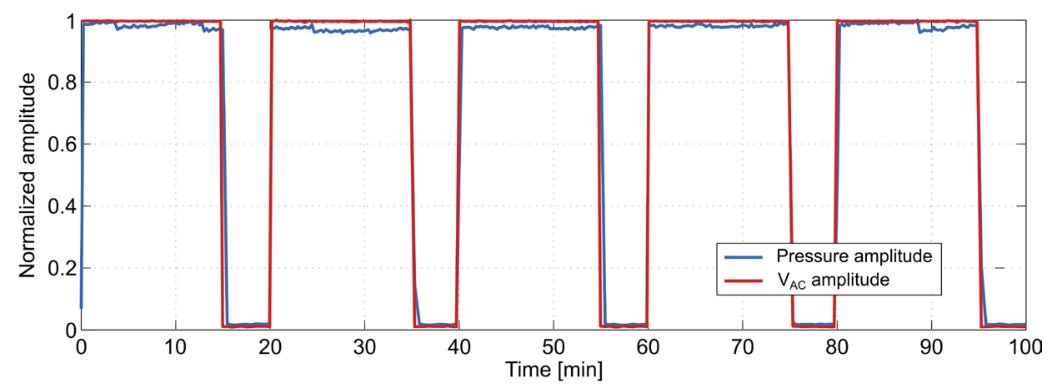

(a)

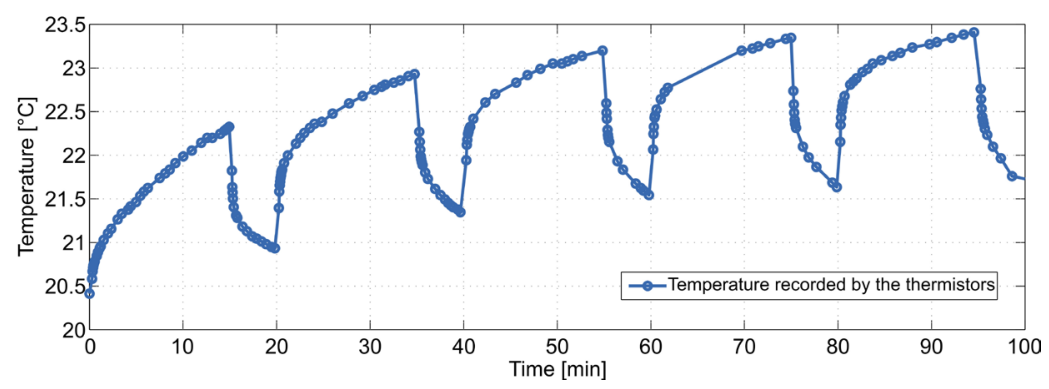

(b)

Figure 9. Variation of the pressure and $V_{A C}$ amplitude over time (a); self-heating of the probe (b). 
No significant shifts of the $V_{A C}$ or pressure amplitudes were observed during the measurement. For instance, the maximum fluctuation of the pressure amplitude is approximately $4 \%$ of the maximal value. Additionally, the self-heating of the probe was circumscribed to approximately $2^{\circ} \mathrm{C}$ during a 15 -minute exposure. Over the entire duration of the experiment, the total temperature variation is approximately $3^{\circ} \mathrm{C}$, as 5 minutes without emission seem not to be enough to totally dissipate the low self-heating.

\section{Discussion}

\subsection{FUS Pressure Field}

The nearly perfect matching between the measured and simulated pressure fields validates by itself the good functionality of all the LF transducers, both focusings provided by the US scanner and the mechanical frame, and the manufacturing of the probe. The coating of silicone rubber, which appeared to be a critical step because of the probe shape, does not seem to impact the CMUT cell behavior. Without the use of electronic focusing, one can nevertheless see a slight asymmetry of the focal spot, showing a small behavioral heterogeneity of the CMUT elements.

Our CMUT-based USgFUS probe can be compared with the piezoelectric-based USgFUS probe reported in [25]. As expected by their sharing a similar design, the shape of the focal spot is roughly the same. The main difference is in the dimension of the mainlobe in depth, which is naturally shorter in our case, as the focusing gain is greater. The sidelobes surrounding the mainlobe are due to the constructive interference of the four LF ultrasonic beams. As the total volume of the focal spot is far smaller than that of the targeted tissues, these sidelobes contribute beneficially to the power deposition. Without electronic focusing, the results are quite the same, except for the size of the azimuth, which then exceeds $10 \mathrm{~mm}$.

Depending on the target applications, the probe exhibits good versatility to tune both the insonified volume and the pressure amplitude. For TSL release, one will focus on the pressure amplitude at the expense of the focal spot. In this case, a PTP pressure of $3 \mathrm{MPa}$ can be achieved, which is in line with the minimum target value defined by [29]. This consideration is in agreement with the temperature elevation measured thereafter, since the TSLs melting temperature had been reached with the target acoustic parameters. For less demanding applications-like sonoporation-a tissue volume of approximately $200 \mathrm{~mm}^{3}$ can be insonified with a maximum PNP pressure of $540 \mathrm{kPa}$. This volume is typically in line with that of common xenograft tumor models. Obviously, all intermediate shades of pressure amplitude and insonified volumes can also be obtained.

Compared with the piezoelectric USgFUS probe, the maximum pressure at the focal point is lower, and achieved at a higher transmit voltage. The total element surface is lower as well, being approximately $75 \%$ of the radiation surface of the piezoelectric-based USgFUS probe. 
Considering the inactive surface between each CMUT cell, the effective radiation surface is $54 \%$ of its piezoelectric counterpart. In future work, a better filling factor should be achieved, and therefore a significant increase of the output pressure is expected. Furthermore, additional progress in the chips homogeneity should enable the use of a more optimized bias voltage and thereby lead to a better transmit sensitivity as well.

The optimal emitting frequency is close to $1 \mathrm{MHz}$, precisely at $1.25 \mathrm{MHz}$ with a maximum PTP pressure of approximately 3.6 MPa. This increase in the PTP pressure amplitude is due to the symmetrization of the signal because of the linearization of the CMUT response with the frequency. Figure 10 reports the harmonic distortion HD3 of the pressure signal, which is defined as

$$
H D_{n}=20 \log _{10}\left(\frac{\sqrt{\sum_{i=2}^{n} A_{i}^{2}}}{A_{1}}\right)
$$

where $A_{i}$ is the signal amplitude of the $i^{\text {th }}$ harmonic component and $n$ is the highest considered harmonic. One can note that although the emitting signals are increasingly distorted with the frequency, the CMUT behavior is increasingly linear. The higher frequencies being closer to the center frequency of the transducers could explain this behavior, as already shown by [37]. Furthermore, the addition of the inductance could linearize the CMUT behavior in a similar way to the harmonic compensation proposed by [38]. This aspect needs to be investigated more deeply.

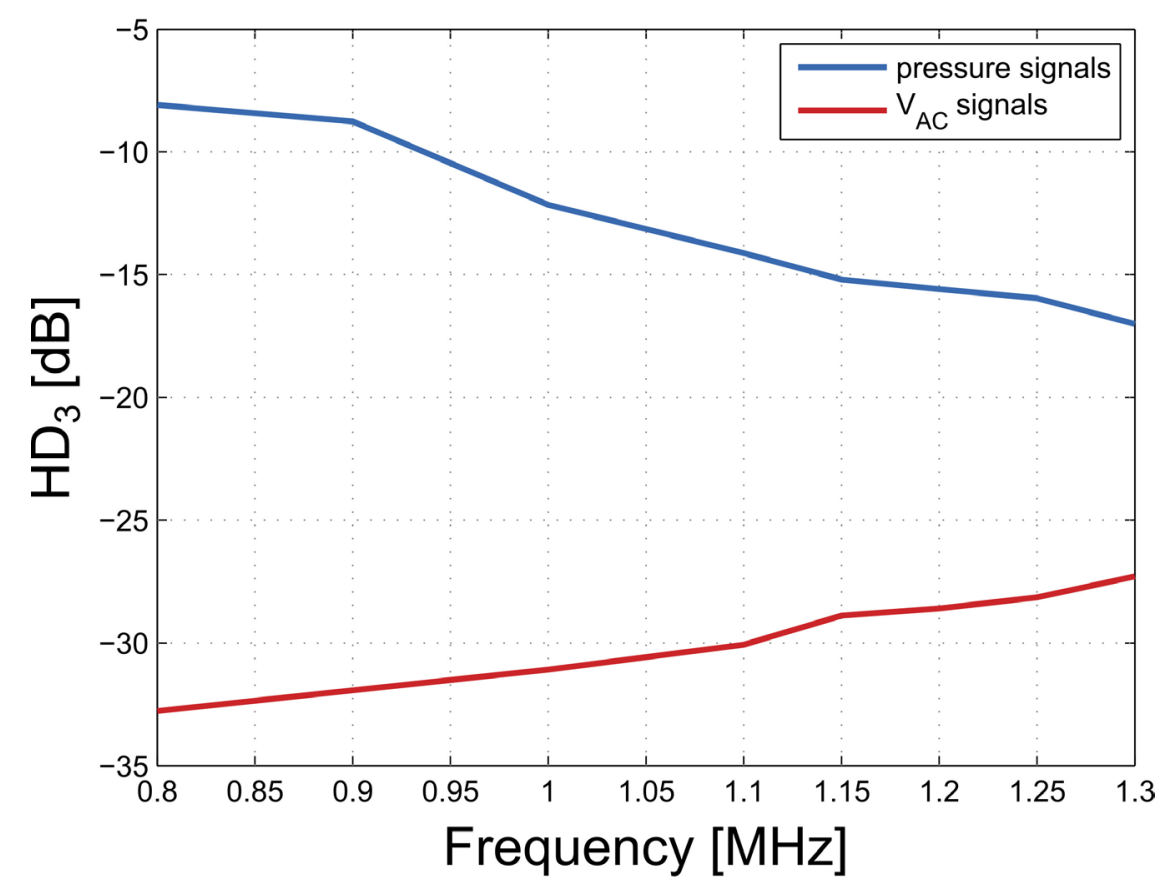

Figure 10. Variation of the harmonic distortion of the pressure and $V_{A C}$ signals for different ultrasonic frequencies. 
Unfortunately, the electronic drivers embedded in the current US scanner are unable to supply the average power required to fire a long duty cycle at this optimal frequency of $1.25 \mathrm{MHz}$. This is why this operating point has not been exploited yet. Nevertheless, we are strongly confident that future improvements of the scanner capabilities will easily lead to significantly better performance.

\subsection{Mild Hyperthemia}

As predicted from the previously obtained results on the pressure field, the CMUT probe is able to induce the temperature elevation required to reach the TSLs melting temperature, at least in our in vitro setup. The maximum temperature elevation is approximately $7.5^{\circ} \mathrm{C}$, which means that one could afford to slightly defocus the US beam to increase the insonified volume. Furthermore, [39] showed that the mean absorption coefficient of human soft tissues is approximately $0.54 \mathrm{~dB} / \mathrm{cm}$ (or $6.2 \mathrm{~Np} / \mathrm{m}$ ) at $1 \mathrm{MHz}$, higher than that of glycerol $(3.6 \mathrm{~Np} /(\mathrm{m} \cdot \mathrm{MHz}))$. This means that even if an in vivo evaluation will introduce thermal dissipation due to blood perfusion, we could still expect good performance on small animals, depending on the insonified tissue.

\subsection{Pseudo-Static Use of CMUT Transducers}

As stated in the description of the probe, CMUT cells were designed to use them at below their resonant frequency in fluid. From the results presented here and the pressure amplitudes radiated by the probe, this approach seems perfectly valid. It would be instructive to continue progress in this direction, especially for the development of multi-frequency ultrasonic devices where the resonant frequency could be used for one purpose and the pseudo-static emission could be exploited for therapeutic modalities. This aspect, which is specific to the CMUT technology, will be more precisely addressed in a forthcoming study.

\subsection{Reliability}

At the current stage of development of the CMUT transducers, reliability considerations are almost as important as the intrinsic acoustic performances. Particularly for therapeutic applications where long burst sequences are fired, the aging of the device is a critical aspect for the technology. For instance, the trapping of charges may occur in the dielectric layers compounding the CMUT transducers, which could lead to a spurious shift in the vibrating operation point. Considering the relatively high pressure and duty cycles involved, this could quickly result in safety issues for the patient. In the results we presented, though, no significant variation in the pressure amplitude is observed.

Conversely, one of the key aspects of the CMUT transducers is the validation of their expected low self-heating. This could prove to be a breakthrough in the field of therapeutic transduction. In the preliminary test that we carried out for 100 minutes, a total temperature elevation of $3^{\circ} \mathrm{C}$ was measured using the embedded thermistors. This is an insignificant rise, and in any case, it would not 
compromise the use of our probe. This result is encouraging, but it needs to be confirmed for longer duty cycles and even in continuous waves. Generally, even if this aspect has already been studied [17] [40], there is still room for a more complete investigation. Future improvements in the maximum duty cycle that the US scanner can sustain are expected.

\section{Conclusions}

This article described the in vitro characterization of an USgFUS CMUT probe for targeted therapy applications, connected to its dedicated US scanner. The different experimental results from this study are all consistent with the target performances and the simulated results. Measurements on xenograft living mice need to be performed to validate the heating capabilities of the probe for TSL release and sonoporation.

The device showed good signs of reliability during demanding excitation bursts. Note that all the results have been obtained on the very first prototype made, which is very satisfactory if we consider the relative level of complexity of the device. This is particularly encouraging for further developments and highlights the potential of the CMUT technology for the development of multi-frequency USgFUS probes.

In this regard, there is plenty of room for improvement in several aspects. Upgrading the current US scanner could provide the possibility to sustain CW therapeutic operation at higher excitation voltages, and adding LF channels could facilitate the electronic focusing of the FUS beam. These improvements could lead to outdating the concave shape of the probe and thereby allow the use of a single monolithic chip embedding all of the LF arrays and the imaging array. The compactness of the device and its exploitation would be greatly facilitated, especially for small animal purposes.

\section{Acknowledgements}

The Agence Nationale de la Recherche and the Fonds Européen de Développement Régional are acknowledged for their financial support on the projects Lab-TMEMS ANR-14-LAB5-0004-01/BRAINMUT 2014-00091557.

The authors want to thank Althaïs Technologies (from the University of Tours) for the development of the US scanner, and J.-M. Grégoire (Inserm Imagerie et Cerveau UMR 930 University of Tours) for his expertise in electronic development. Our acknowledgments are also given to the engineering and electronics staff of Vermon for the probe conception, as well as to N. Sénégond, T. Matéo, E. Kanbar and J. Heller for their kind assistance.

\section{References}

[1] Kim, H.J., Greenleaf, J.F., Kinnick, R.R., Bronk, J.T. and Bolander, M.E. (1996) Ultrasound-Mediated Transfection of Mammalian Cells. Human Gene Therapy, 7, 1339-1346. https://doi.org/10.1089/hum.1996.7.11-1339

[2] van Wamel, A., Kooiman, K., Harteveld, M., Emmer, M., ten Cate, F.J., Versluis, M. 
and de Jong, N. (2006) Vibrating Microbubbles Poking Individual Cells: Drug Transfer into Cells via Sonoporation. Journal of Controlled Release, 112, 149-155. https://doi.org/10.1016/j.jconrel.2006.02.007

[3] Levy, D., Kost, J., Meshulam, Y. and Langer, R. (1989) Effect of Ultrasound on Transdermal Drug Delivery to Rats and Guinea Pigs. Journal of Clinical Investigation, 83, 2074-2078. https://doi.org/10.1172/JCI114119

[4] Hynynen, K., McDannold, N., Sheikov, N.A., Jolesz, F.A. and Vykhodtseva, N. (2005) Local and Reversible Blood-Brain Barrier Disruption by Noninvasive Focused Ultrasound at Frequencies Suitable for Trans-Skull Sonications. Neuroimage, 24, 12-20. https://doi.org/10.1016/j.neuroimage.2004.06.046

[5] Mitragotri, S. (2005) Healing Sound: The Use of Ultrasound in Drug Delivery and Other Therapeutic Applications. Nature Reviews Drug Discovery, 4, 255-260. https://doi.org/10.1038/nrd1662

[6] Cline, H.E., Schenck, J.F., Hynynen, K., Watkins, R.D., Souza, S.P. and Jolesz, F.A. (1992) MR-Guided Focused Ultrasound Surgery. Journal of Computer Assisted Tomography, 16, 956-965. https://doi.org/10.1097/00004728-199211000-00024

[7] Shen, S.H., Fennessy, F., McDannold, N., Jolesz, F. and Tempany, C. (2009) Image-Guided Thermal Therapy of Uterine Fibroids. Seminars in Ultrasound, CT and MRI, 30, 91-104. https://doi.org/10.1053/j.sult.2008.12.002

[8] Ebbini, E.S., Yao, H. and Shrestha, A. (2006) Dual-Mode Ultrasound Phased Arrays for Image-Guided Surgery. Ultrasonic Imaging, 28, 65-82.

https://doi.org/10.1177/016173460602800201

[9] Haller, M.I. and Khuri-Yakub, P. (1994) A Surface Micromachined Electrostatic Ultrasonic Air Transducer. IEEE Transactions on Ultrasonics, Ferroelectrics, and Frequency Control, 43, 1-6. https://doi.org/10.1109/ULTSYM.1994.401810

[10] Oralkan, O., Ergun, A.S., Johnson, J.A., Karaman, M., Demirci, U., Kaviani, K., Lee, T.H. and Khuri-Yakub, B.T. (2002) Capacitive Micromachined Ultrasonic Transducers: Next-Generation Arrays for Acoustic Imaging? IEEE Transactions on UItrasonics, Ferroelectrics, and Frequency Control, 49, 1596-1610. https://doi.org/10.1109/TUFFC.2002.1049742

[11] Mills, D. and Smith, L. (2003) Real-Time In-Vivo Imaging with Capacitive Micromachined Ultrasound Transducer (cMUT) Linear Arrays. IEEE Symposium on UItrasonics, 1, 568-571. https://doi.org/10.1109/ULTSYM.2003.1293467

[12] Oralkan, O., Hansen, S., Bayram, B., Yaralioglu, G., Ergun, A. and Khuri-Yakub, B. (2004) High-Frequency CMUT Arrays for High-Resolution Medical Imaging. IEEE Ultrasonics Symposium, 1, 399-402. https://doi.org/10.1109/ULTSYM.2004.1417747

[13] Yeh, D.T., Oralkan, O., Wygant, I.O., O’Donnell, M. and Khuri-Yakub, B.T. (2006) Ultrasound Imaging using a Forward-Looking CMUT Ring Array for Intravascular/Intracardiac Applications. IEEE Transactions on Ultrasonics, Ferroelectrics, and Frequency Control, 53, 1202-1210. https://doi.org/10.1109/TUFFC.2006.1642519

[14] Savoia, A.S., Caliano, G. and Pappalardo, M. (2012) A CMUT Probe for Medical Ultrasonography: From Microfabrication to System Integration. IEEE Transactions on Ultrasonics, Ferroelectrics and Frequency Control, 59, 1127-1138. https://doi.org/10.1109/TUFFC.2012.2303

[15] Christiansen, T.L., Rasmussen, M.F., Bagge, J.P., Moesner, L.N., Jensen, J.A. and Thomsen, E.V. (2015) 3-D Imaging using Rowcolumn-Addressed Arrays with Integrated Apodization Part II: Transducer Fabrication and Experimental Results. IEEE 
Transactions on Ultrasonics, Ferroelectrics, and Frequency Control, 62, 959-971. https://doi.org/10.1109/TUFFC.2014.006819

[16] Zhao, D., Zhuang, S. and Daigle, R. (2015) A Commercialized High Frequency CMUT Probe for Medical Ultrasound Imaging. IEEE International Ultrasonics Symposium (IUS), Taipei, 21-24 October 2015, 1-4. https://doi.org/10.1109/ULTSYM.2015.0063

[17] Wong, S.H., Kupnik, M., Butts-Pauly, K. and Khuri-Yakub, B.T. (2007) Advantages of Capacitive Micromachined Ultrasonics Transducers (CMUTs) for High Intensity Focused Ultrasound (HIFU). IEEE Ultrasonics Symposium Proceedings, New York, 28-31 October 2007, 1313-1316. https://doi.org/10.1109/ULTSYM.2007.330

[18] Wong, S.H., Watkins, R.D., Kupnik, M., Pauly, K.B. and Khuri-Yakub, B.T. (2008) Feasibility of MR-Temperature Mapping of Ultrasonic Heating from a CMUT. IEEE Transactions on Ultrasonics, Ferroelectrics, and Frequency Control, 55, 811-817. https://doi.org/10.1109/TUFFC.2008.715

[19] Wong, S.H., Kupnik, M., Watkins, R.D., Butts-Pauly, K. and Khuri-Yakub, B.T. (2010) Capacitive Micromachined Ultrasonic Transducers for Therapeutic Ultrasound Applications. IEEE Transactions on Biomedical Engineering, 57, 114-123. https://doi.org/10.1109/TBME.2009.2026909

[20] Yamaner, F.Y., Olcum, S., Ouz, H.K., Bozkurt, A., Koymen, H. and Atalar, A. (2012) High-Power CMUTs: Design and Experimental Verification. IEEE Transactions on Ultrasonics, Ferroelectrics, and Frequency Control, 59, 1276-1284. https://doi.org/10.1109/TUFFC.2012.2318

[21] Yoon, H.-S., Chang, C., Jang, J.H., Bhuyan, A., Choe, J.W., Nikoozadeh, A., Watkins, R.D., Stephens, D.N., Butts Pauly, K. and Khuri-Yakub, B.T. (2016) EX Vivo HIFU Experiments using a 32x32-Element CMUT Array. IEEE Transactions on Ultrasonics, Ferroelectrics, and Frequency Control, 63, 2150-2158. http://ieeexplore.ieee.org/document/7560642/

[22] Park, K.K., Lee, H.J., Crisman, P., Kupnik, M., Oralkan, O. and Khuri-Yakub, B.T. (2008) Optimum Design of Circular CMUT Membranes for High Quality Factor in Air. Ultrasonics Symposium, Beijing, 2-5 November 2008, 504-507. https://doi.org/10.1109/TUFFC.2012.2318

[23] Stephens, D.N., Kruse, D.E., Ergun, A.S., Barnes, S., Lu, X.M. and Ferrara, K.W. (2008) Efficient Array Design for Sonotherapy. Physics in Medicine and Biology, 53, 3943-3969. https://doi.org/10.1088/0031-9155/53/14/014

[24] Lai, C.-Y., Kruse, D.E., Caskey, C.F., Stephens, D.N., Sutcliffe, P.L. and Ferrara, K.W. (2010) Noninvasive Thermometry Assisted by a Dual-Function Ultrasound Transducer for Mild Hyperthermia. IEEE Transactions on Ultrasonics, Ferroelectrics and Frequency Control, 57, 2671-2684. https://doi.org/10.1109/TUFFC.2010.1741

[25] Kruse, D., Lai, C.-Y., Stephens, D., Sutcliffe, P., Paoli, E., Barnes, S. and Ferrara, K. (2010) Spatial and Temporal-Controlled Tissue Heating on a Modified Clinical Ultrasound Scanner for Generating Mild Hyperthermia in Tumors. IEEE Transactions on Biomedical Engineering, 57, 155-166. https://doi.org/10.1109/TBME.2009.2029703

[26] Wan, Y. and Ebbini, E.S. (2008) Imaging with Concave Large-Aperture Therapeutic Ultrasound Arrays using Conventional Synthetic-Aperture Beamforming. IEEE Transactions on Ultrasonics, Ferroelectrics, and Frequency Control, 55, 1705-1718. https://doi.org/10.1109/TUFFC.2008.856 
[27] Gross, D., Coutier, C., Legros, M., Bouakaz, A. and Certon, D. (2015) A cMUT Probe for Ultrasound-Guided Focused Ultrasound Targeted Therapy. IEEE Transactions on Ultrasonics, Ferroelectrics, and Frequency Control, 62, 1145-1160. https://doi.org/10.1109/TUFFC.2014.006887

[28] Al Sabbagh, C., Tsapis, N., Novell, A., Calleja-Gonzalez, P., Escoffre, J.-M., Bouakaz, A., Chacun, H., Denis, S., Vergnaud, J., Gueutin, C. and Fettal, E. (2014) Formulation and Pharmacokinetics of Thermosensitive Stealth R Liposomes Encapsulating 5-Fluorouracil. Pharmaceutical Research, 32, 1585-1603.

[29] Novell, A., Al Sabbagh, C., Escoffre, J.-M., Gaillard, C., Tsapis, N., Fattal, E. and Bouakaz, A. (2015) Focused Ultrasound Influence on Calcein-Loaded Thermosensitive Stealth Liposomes. International Journal of Hyperthermia, 31, 349-358. https://doi.org/10.3109/02656736.2014.1000393

[30] Stephens, D.N., Lu, X.M., Proulx, T., Walters, W., Dayton, P., Tartis, M., Kruse, D.E., Lum, A.F.H., Kitano, T., Stieger, S.M. and Ferrara, K. (2006) Multifrequency Array Development for Drug Delivery Therapies: Characterization and First Use of a Triple Row Ultrasound Probe. IEEE Ultrasonics Symposium. Vancouver, 3-6 October 2006, 66-69. https://doi.org/10.1109/ULTSYM.2006.30

[31] Senegond, N., Boulme, A., Plag, C., Teston, F. and Certon, D. (2013) Fast Time-Domain Modeling of Fluid-Coupled cMUT Cells: From the Single Cell to the 1-D Linear Array Element. IEEE Transactions on Ultrasonics, Ferroelectrics, and Frequency Control, 60, 1505-1518. https://doi.org/10.1109/TUFFC.2013.2723

[32] Certon, D., Legros, M., Gross, D., Vince, P., Gens, F., Gregoire, J., Coutier, C., Novell, A. and Bouakaz, A. (2014) Ultrasound Pre-Clinical Platform for Diagnosis and Targeted Therapy. IEEE International Ultrasonics Symposium, Chicago, 3-6 September 2014, 329-332. https://doi.org/10.1109/ULTSYM.2014.0081

[33] Piwakowski, B. (1989) Method for Computing Spatial Pulse Response: Time-Domain Approach. The Journal of the Acoustical Society of America, 86, 2422. https://doi.org/10.1121/1.398449

[34] Piwakowski, B. and Sbai, K. (1999) A New Approach to Calculate the Field Radiated from Arbitrarily Structured Transducer Arrays. IEEE Transactions on Ultrasonics, Ferroelectrics and Frequency Control, 46, 422-440. https://doi.org/10.1109/58.753032

[35] Lingvall, F. (2004) Time-Domain Reconstruction Methods for Ultrasonic Array Imaging-A Statistical Approach. PhD Dissertation, Uppsala University, Uppsala.

[36] Mannaris, C., Efthymiou, E., Meyre, M.E. and Averkiou, M.A. (2013) In Vitro Localized Release of Thermosensitive Liposomes with Ultrasound-Induced Hyperthermia. Ultrasound in Medicine and Biology, 39, 2011-2020.

https://doi.org/10.1109/58.753032

[37] Lohfink, A. and Eccardt, P.-C. (2005) Linear and Nonlinear Equivalent Circuit Modeling of CMUTs. IEEE Transactions on Ultrasonics, Ferroelectrics and Frequency Control, 52, 2163-2172. https://doi.org/10.1109/TUFFC.2005.1563260

[38] Satir, S. and Degertekin, F.L. (2012) Harmonic Reduction in Capacitive Micromachined Ultrasonic Transducers by Gap Feedback Linearization. IEEE Transactions on Ultrasonics, Ferroelectrics, and Frequency Control, 59, 50-59. https://doi.org/10.1109/TUFFC.2012.2155

[39] Mast, T.D. (2000) Empirical Relationships between Acoustic Parameters in Human Soft Tissues. Acoustics Research Letters Online, 1, 37. http://scitation.aip.org/content/asa/journal/arlo/1/2/10.1121/1.1336896 
[40] Ergun, A.S., Barnes, S. and Gardner, E. (2007) 5F-5 An Assessment of the Thermal Efficiency of Capacitive Micromachined Ultrasonic Transducers. Ultrasonics Symposium, New York, 28-31 October 2007, 420-423.

https://doi.org/10.1109/ULTSYM.2007.114 